Departamento de Medicina Interna, Facultad de Medicina Pontificia Universidad Católica de Chile.

anterno de Medicina Universidad Austral de Chile.

bInterna de Medicina, Pontificia Universidad Católica de Chile. 'Residente de Geriatría, Pontificia Universidad Católica de Chile.

Recibido el 7 de marzo de 2014 aceptado el 10 de septiembre de 2014

Correspondencia a: Dr. Gonzalo Eymin Hospital Clínico Pontificia Universidad Católica de Chile. Marcoleta $367,5^{\circ}$ piso, Santiago, Chile. Teléfono: 56-2-23543151 geymin@med.puc.c

\section{Hemólisis, exantema, serositis. Manifestaciones extrapulmonares del Mycoplasma pneumoniae. Reporte de un caso}

\author{
ROBERTO MONDACA P.a, VICTORIA PIZARRO C. ${ }^{\mathrm{b}}$, \\ VÍCTOR CARES', GONZALO EYMIN
}

\section{Hemolysis, serositis and exanthema induced by Mycoplasma pneumoniae infection. Report of one case}

\begin{abstract}
Mycoplasma infections have extrapulmonary manifestations that may be associated with respiratory symptoms and may have skin, heart, gastrointestinal, rheumatologic, neurologic, hematologic involvement. Cold agglutinin mediated autoimmune hemolytic anemia is the most common hematological manifestation. We report a 27-year-old woman infected with Mycoplasma pneumoniae, who presented respiratory involvement with pneumonia, exanthema, serositis and acute hemolytic anemia that required transfusion. The key for the diagnosis were the extrapulmonary manifestations associated with respiratory involvement after five days of hospitalization.
\end{abstract}

(Rev Med Chile 2014; 142: 1334-1337)

Key words: Exanthema; Hemolysis; Mycoplasma pneumoniae; Serositis.
E n la infección por Mycoplasma pneumoniae el compromiso pulmonar es lo más frecuente de observar; sin embargo, ésta puede presentarse como un cuadro multisistémico, con manifestaciones cutáneas, hematológicas, neurológicas, cardiacas, gastrointestinales, reumatológicas, entre otras. La aparición de crioaglutininas es lo más característico dentro del espectro hematológico, lo cual no siempre se acompaña de hemólisis clínica. A continuación se presenta el caso de una mujer con compromiso respiratorio en que la hemólisis y el exantema dieron las claves para sospechar infección por Mycoplasma pneumoniae.

\section{Caso clínico}

Mujer de 27 años, con antecedentes de hipotiroidismo, dudoso síndrome antifosfolípidos y tromboembolismo pulmonar (TEP) en tratamiento con acenocumarol. Consulta por cuadro de 3 h de evolución caracterizado por dolor sordo, de inicio insidioso, intenso, localizado en parte baja de tórax e hipocondrio derecho, asociado a compromiso del estado general y diaforesis. Al examen físico destacaba taquicardia de 100 por minuto, normotensión, frecuencia respiratoria de 20 por minuto, temperatura de $36^{\circ}$, sudoración, palidez, exantema morbiliforme en dorso, examen pulmonar normal, y sensibilidad abdominal difusa, sin signos de irritación peritoneal. Examen neurológico normal. Los exámenes al ingreso mostraron hematocrito 32,9\%, hemoglobina 11,6 $\mathrm{g} / \mathrm{dl}, \mathrm{VCM} 88 \mathrm{fl}$, leucocitos $11.400 / \mathrm{mm}^{3}$, plaquetas $311.000 / \mathrm{mm}^{3}$, VHS $65 \mathrm{~mm} / \mathrm{h}$, BUN $8 \mathrm{mg} / \mathrm{dl}$, creatinina $1,16 \mathrm{mg} / \mathrm{dl}$, bilirrubina $0,43 \mathrm{mg} / \mathrm{dl}$ (normal), LDH $148 \mathrm{mg} / \mathrm{dl}$ (normal <225), PCR 3,06 mg/dl (normal $<0,5)$. Se realizó un AngioTAC de tórax que descartó TEP, mostrando áreas con densidad en vidrio esmerilado bibasales por lo que se inició ceftriaxona. En los cortes de abdomen y pelvis se encontró moderada ascitis en excavación pelviana.

Al día siguiente se evidenció caída del hemato- 
crito a $24,8 \%$, y al día subsiguiente a $19,6 \%$, con recuento de reticulocitos de $1,5 \%$, sin alteración de las otras series ni evidencias de sangrado. Dado que paciente estaba sintomática, se transfundieron 2 unidades de hematíes y se realizó una endoscopia digestiva alta que resultó normal. Ese mismo día paciente presentó desaturación hasta $89 \%$ y fiebre de $38^{\circ} \mathrm{C}$. Se realizó TAC de tórax con contraste que evidenció progresión de los focos de condensación y derrame pleural bilateral laminar. Se planteó cuadro de neumonía por germen atípico, y se cambió terapia a levofloxacino.

Dado el aumento de LDH hasta $465 \mathrm{mg} / \mathrm{dl}$, la reticulocitosis ( $4,5 \%$ al control), y la presencia de sangre en la orina completa en el análisis químico, pero no en el físico, se planteó hemólisis. Se solicitó haptoglobina y complemento que resultaron normales y Coombs directo que fue negativo. Dado la presencia de neumonía, evidencias de hemólisis con Coombs negativo, exantema y ascitis, se plantea infección por Mycoplasma. Se solicitó crioaglutininas e IgM para Mycoplasma resultando ambas positivas (Crioaglutininas 1/128). La paciente completó 14 días con levofloxacino evolucionando asintomática, con examen físico normal, y con hemograma que evidenció hematocrito de $35,9 \%$, hemoglobina $12,3 \mathrm{~g} / \mathrm{dl}$, leucocitos $5.300 /$ $\mathrm{mm}^{3}$, plaquetas $351.000 / \mathrm{mm}^{3}$, VHS $60 \mathrm{~mm} / \mathrm{h}$, PCR 0,76 mg/dl (peak 11,13), LDH $213 \mathrm{mg} / \mathrm{dl}$.

Se concluye que cuadro fue compatible con anemia hemolítica autoinmune por crioaglutininas, neumonía y rash secundarios a infección por Mycoplasma, probablemente gatillada por la infusión de soluciones salinas a temperatura ambiente.

\section{Discusión}

Se presenta el caso de una mujer con neumonía adquirida en la comunidad por Mycoplasma que presentó escasa sintomatología respiratoria, predominando manifestaciones extrapulmonares como el exantema, la serositis y la hemólisis.

La infección por Mycoplasma es asintomática la mayor parte de las veces, siendo el sistema respiratorio el afectado con mayor frecuencia ${ }^{1}$. En la serie de Mansel, 75-100\% de los pacientes desarrolló tos no productiva intratable y sólo 3-10\% desarrolló neumonía ${ }^{2}$. El dolor pleurítico es raro $^{3}$ y la tos es lo más frecuente ${ }^{4}$. En la neumonía el examen pulmonar puede ser normal y no existir hallazgos que orienten al diagnóstico ${ }^{5}$. La radiografía de tórax puede mostrar hallazgos inespecíficos o patrón retículo nodular uni o bilateral y áreas de condensación difusas bibasales ${ }^{6}$. La TAC puede mostrar opacidades irregulares nodulares y centrolobulillares, en vidrio esmerilado, condensación y engrosamiento del paquete broncovascular ${ }^{7}$. En $15-20 \%$ de los casos se encuentra derrame pleural ${ }^{2}$.

El compromiso extrapulmonar de la infección por Mycoplasma no debe olvidarse, ya que puede dar la clave cuando se asocian a clínica respiratoria, destacando las manifestaciones cutáneas, cardiacas, gastrointestinales, reumatológicas, neurológicas, hematológicas, etc. En la Tabla 1 se resumen dichas manifestaciones ${ }^{8-18}$.

La sensibilidad de las serologías de detección de Mycoplasma depende de cuando se toman en relación a la aparición de síntomas, y de la disponibilidad de IgG seriadas. La IgM suele aparecer después de 7 días de iniciada la infección y alcanza su máximo a las 3 a 6 semanas, sin embargo, en adultos la presencia de IgM puede no traducir infección reciente, $y$, por otro lado, $20 \%$ de los adultos no montan respuesta de IgM ante una infección reciente ${ }^{19-20}$. En relación a la $\mathrm{IgG}$, un aumento significativo (4 veces) de los niveles a las 2 a 3 semanas también traduciría infección. En la reinfección no hay respuesta de $\operatorname{IgM}$, sino un rápido incremento de los niveles de $\operatorname{IgG}^{20}$. El estudio de Busson que analizó 4 técnicas de detección de IgM en 120 pacientes (Mediana de 15 años) mostró una sensibilidad de 100\% y una especificidad entre $68 \%$ y $80 \%$ (Muestras seriadas $)^{21}$. La técnica empleada en nuestro laboratorio (IgM Elisa) tiene una sensibilidad y especificidad de 89,1 y $92,2 \%$ respectivamente. Nilsson comparó la sensibilidad de la PCR de secreciones respiratorias versus la detección serológica (IgG e IgM) en pacientes con infección respiratoria por Mycoplasma, destacando que la sensibilidad durante la primera semana del inicio de síntomas fue de $100 \%$ para la PCR y $23 \%$ para la serología ${ }^{22}$. La presencia de falsos positivos para IgM se puede ver en cuadros producidos por virus de Ebstein Barr, Citomegalovirus y Coronavirus. También se pueden ver valores intermedios de IgG e IgM en casos de Sarampión, Virus respiratorio sincicial, Virus Varicela zoster y Chlamidea pneumoniae ${ }^{22}$. En casos dudosos se recomienda la determinación de crioaglutininas. Dado que más de la mitad de los pacientes con infección por Mycoplasma presentan crioaglutininas, la presencia de títulos 
Hemólisis, serositis y exantema inducidos por infección por Mycoplasma pneumoniae - R. Mondaca et al

Tabla 1 Manifestaciones extrapulmonares de la infección por Mycoplasma pneumoniae

\begin{tabular}{|lll|}
\hline Cutáneas & Rash máculo papular eritematoso & $8 \%-33 \%$ \\
& Síndrome Stevens-Johnson & $1 \%-5 \%$ \\
Gastrointestinales & Náuseas, vómitos, anorexia o diarrea & $45 \%$ \\
& Pancreatitis y hepatitis & $\mathrm{NR}$ \\
Cardíacas & $\begin{array}{l}\text { Inversión de la onda T, bradicardia, prolongación } \\
\text { del PR y QRS estrecho }\end{array}$ & $\mathrm{NR}$ \\
& Miocarditis aguda, pericarditis y miopericarditis & $<5 \%$ \\
Neurológicas & $\begin{array}{l}\text { Encefalitis, meningoencefalitis, polirradiculitis, meningitis aséptica, } \\
\text { ataxia cerebelosa, psicosis parálisis de nervios craneales, mielitis }\end{array}$ & $7 \%$ de hospitalizados \\
Hematológicas & transversa, Guillain Barre & \\
& $\begin{array}{l}\text { Anemia hemolítica, coagulación intravascular diseminada } \\
\text { Púrpura trombocitopénica trombótica, anemia aplástica, Raynaud, }\end{array}$ & Reportes aislados \\
& trombosis arterial & \\
Osteomusculares & Artralgias, mialgias, poliartropatías & $14 \%$ \\
& Rabdomiolisis & 8 reportados. 7 en niños \\
\hline Otras & Otitis media aguda, glomérulonefritis, uveítis anterior & Reportes aislados \\
\hline
\end{tabular}

NR: No reportado.

$>1: 32$ es altamente sugerente de infección. Éstas son autoanticuerpos de tipo IgM que aglutinan eritrocitos en un rango entre los $4^{\mathrm{o}}$ y $37^{2,3}$. La mayoría de las veces esta hemólisis no se traduce en manifestaciones clínicas importantes ${ }^{2}$, sin embargo, en casos con títulos altos la hemólisis puede ser severa ${ }^{18}$. En general el cuadro hemolítico aparece durante la segunda a tercera semana posterior al inicio de la fiebre ${ }^{23}$. El pronóstico de la enfermedad suele ser benigno, y la hemólisis remite generalmente a las 4-6 semanas ${ }^{18}$. La sospecha de hemólisis se sustenta principalmente en el aumento de $\mathrm{LDH}$, hiperbilirrubinemia indirecta, reticulocitosis y haptoglobina baja, orientándonos además en este caso el hallazgo de sangre en el análisis químico de orina, pero no en el físico, lo que sugiere pigmenturia. La observación al frotis de eritrocitos aglutinados sugiere reacción mediada por crioaglutininas. El test de Coombs directo suele ser negativo debido a que la hemólisis es mediada principalmente por activación del complemento ${ }^{18}$. En el diagnóstico diferencial debe considerarse los linfomas, infecciones por virus de Ebstein Barr, Citomegalovirus, Influenza, Varicella, y por bacterias como Legionella y Citrobacter ${ }^{24}$. La transfusión de hemoderivados calentados a $37^{\circ}$ podría disminuir el riesgo de hemólisis ${ }^{25}$.

Nuestra paciente acude a urgencias con tope inspiratorio y dolor en hipocondrio derecho. Dado sus antecedentes, lo primero que se descartó fue TEP. La aparición de anemia aguda fue el eje que motivó su estudio diagnóstico y manejo. Al quinto día de hospitalización, se planteó que el cuadro era secundario a infección por Mycoplasma dado la aparición de infiltrados pulmonares, exantema, anemia aguda y pigmenturia. Si bien no encontramos reportes en la literatura de ascitis sin hepatitis como manifestación extrapulmonar de infección por Mycoplasma, en la TAC de control ésta ya había desaparecido, lo cual sugiere que haya sido un epifenómeno, o bien una manifestación extrapulmonar no descrita. Los niveles normales de haptoglobina pueden explicarse por la infección, lo cual induciría su producción como reactante de fase aguda.

\section{Conclusión}

La infección por Mycoplasma pneumoniae puede tener variadas presentaciones y afectar a diversos sistemas. La presencia de sintomatología respiratoria asociada a exantema, manifestaciones hematológicas, neurológicas u otras, obliga a plantear una infección por Mycoplasma pneumoniae. Esto es fundamental si consideramos que en nuestro medio no es infrecuente que las neumonías se traten con antibióticos beta-lactámicos, los cuales dejan sin cobertura a este organismo que carece 
de pared celular. La anemización inexplicada en un paciente hospitalizado obliga a considerar hemólisis por anticuerpos en frío. Esto se debe a que las crioaglutininas tienen un rango térmico de aglutinación que es variable $\left(4^{\circ}\right.$ a $\left.37^{\circ}\right)$, y por lo tanto, la hemólisis puede gatillarse al pasar sueros a temperatura ambiente. Si bien la mayor parte de las veces la hemólisis es de poca cuantía, esta puede ser intensa, llevando a anemización importante que requiera transfusión como fue el caso de nuestra paciente. Ante títulos indeterminados de IgGo de IgM la presencia de crioaglutininas aumenta la posibilidad de infección por Mycoplasma. En casos sospechosos con IgM negativa la determinación de PCR en secreciones respiratorias puede ser una alternativa diagnóstica más sensible.

\section{Referencias}

1. Luby JP. Pneumonia caused by Mycoplasma pneumoniae infection. Clin Chest Med 1991; 12: 237.

2. Mansel JK, Rosenow EC 3rd, Smith TF, Martin JW Jr. Mycoplasma pneumoniae pneumonia. Chest 1989; 95: 639.

3. Taylor-Robinson D. Infections due to species of Mycoplasma and Ureaplasma: an update. Clin Infect Dis 1996; 23: 671.

4. Marrie TJ, Beecroft M, Herman-Gnjidic Z, PoulinCostello M. Symptom resolution in patients with $\mathrm{Myco}$ plasma pneumoniae pneumonia. Can Respir J 2004; 11 (8): 573-7.

5. Clyde WA Jr. Clinical overview of typical Mycoplasma pneumoniae infections. Clin Infect Dis 1993; 17 Suppl 1: S32.

6. Finnegan OC, Fowles SJ, White RJ. Radiographic appearances of mycoplasma pneumonia. Thorax 1981; 36: 469-72.

7. Miyashita N, Akaike H, Teranishi H, Nakano T, Ouchi K, Okimoto N. Chest computed tomography for the diagnosis of Mycoplasma pneumoniae infection. Respirology 2014; 19: 144-5.

8. Schalock PC, Dinulos JGH. Mycoplasma pneumoniaeinduced cutaneous disease. International Journal of Dermatology 2009; 48: 673-81.

9. Ficko C, Mellon G, Andriamanantena D, Merens A, Rapp C. Acute Mycoplasma pneumonia pancreatitis. Med Mal Infect 2011; 41 (11): 617-8.

10. Shin S, Park S, Kim J, Ha J, Kim Y, Jung S, Kim J, Lee M, Park C. Clinical characteristics of patients with $\mathrm{Myco-}$ plasma pneumoniae-related acute hepatitis. Digestion
2012; 86 (4): 302-8

11. Nalmas S, Nagarakanti R, Slim J, Abter E, Bishburg E. Electrocardiographic Changes in Infectious Diseases. Hospital Physician 2007; 9: 15-27.

12. Paz A, Potasman I. Mycoplasma-associated carditis: case reports and review. Cardiology 2002; 97: 83-8.

13. Ponka A. Carditis associated with Mycoplasma pneumoniae infection. Acta Med Scand 1979; 206: 77-86.

14. Guleira R, Nisar N, Chwla TC, Bismas NR. Mycoplasma pneumoniae and central nervous system complications: a review. J Lab Clin Med 2005; 146: 55-63.

15. Vervloet LA, Marguet C, Camargos PA. Infection by Mycoplasma pneumoniae and its importance as an etiological agent in childhood community acquired pneumonias. Braz J Infect Dis 2007; 11: 507-14.

16. Waites KB, Talkington DF. Mycoplasma pneumonia and its role as a human pathogen. Clinical Microbiology Reviews, vol. 17, nº 4, pp. 697-728, 2004.

17. Vitullo BB, O’Regan S, de Chadarevian JP, Kaplan BS. Mycoplasma pneumonia associated with acute glomerulonephritis. Nephron 1978; 21 (5): 284.

18. Berentsen S, Tjonnfjord GE. Diagnosis and treatment of cold agglutinin mediated autoimmune hemolytic anemia. Blood Rev 2012; 26: 107-15.

19. Thacker WL, Talkington DF. Analysis of complement fixation and commercial enzyme immunoassays for detection of antibodies to Mycoplasma pneumonia in human serum. Clin Diagn Lab Immunol 2000; 7 (5): 778-80.

20. Zhang L, Zong ZY, Liu YB, Ye H, Lv XJ. PCR versus serology for diagnosing Mycoplasma pneumoniae infection: a systematic review \& meta-analysis. Indian J Med Res 2011; 134: 270-80.

21. Busson L, Van den Wijngaert S, Dahma H, Decolvenaer M, Di Cesare L, Martin A, et al. Evaluation of 10 serological assays for diagnosing Mycoplasma pneumoniae infection. Diagn Microbiol Infect Dis 2013; 76 (2): 1337.

22. Nilsson AC, Björkman P, Persson K. Polymerase chain reaction is superior to serology for the diagnosis of acute Mycoplasma pneumoniae infection and reveals a high rate of persistent infection. BMC Microbiol 2008; 8: 93.

23. Dacie J. Auto-immune haemolytic anaemia (AIHA): cold-antibody syndromes III: haemolytic anaemia following mycoplasma pneumonia. In: Dacie J, editor. The Haemolytic Anaemias, Vol. 3. London: Churchill Livingstone; 1992. p. 296-312.

24. Swiecicki PL, Hegerova LT, Gertz MA. Cold agglutinin disease. Blood 2013; 122 (7): 1114.

25. Gertz MA. Cold hemolytic syndrome. Hematology Am Soc Hematol Educ Program 2006: 19-23. 